\title{
Robust control for aircraft anti-skid braking system based on dynamic tire/road friction force model
}

\author{
Li Fengyu \\ School of Automation Science and Electrical Engineering \\ Beijing University of Aeronautics and Astronautics \\ Beijing, China \\ alexander.lee1983@gmail.com
}

\author{
Jiao Zongxia \\ School of Automation Science and Electrical Engineering \\ Beijing University of Aeronautics and Astronautics \\ Beijing, China \\ zxjiao@buaa.edu.cn
}

\begin{abstract}
The aircraft anti-skid braking system plays an important role for the safety during taxiing. Aircraft tire/road friction force is essential information for model based control law. In this paper, a robust control strategy with an observerbased dynamic aircraft tire/road model is given to improve the brake quality. Firstly, the nonlinear fuselage and wheel models are derived. The tire/road friction force is derived with LuGre dynamic friction model considering velocity correlation, in which the internal friction state is estimated by dual-observer. In case of external disturbance and modeling error, a robust controller is employed to track the online calculated desired slip rate. The simulation results show that the well-designed controller has stable brake performance.
\end{abstract}

Keywords-aircraft braking control; LuGre dynamic friction model; robust control; state estimation; nonlinear control

\section{INTRODUCTION}

The reliability of the aircraft anti-skid braking system plays an important role for the safety of passengers and airports, which can stop the aircraft smoothly and effectively. When applying pressure on aircraft anti-skid braking system (ABS) during taking off, landing and taxing, high efficiency control strategy is needed to ensure the aircraft ground safety. The aircraft braking system is a complex nonlinear system with uncertainties. The nonlinearities of system are constructed by the nonlinear relationship between aircraft tire and runny (or road) adhesion coefficient, adhesion coefficient and slip rate, and brake torque and pressure ${ }^{[1]}$. It is rather difficult to get an accurate braking system and tire/road friction models and their parameters. Thus, nonlinear control theory is introduced to solve the problem mentioned above.

In the past, many kinds of control strategies were utilized in simulations, hardware-in-the-loop (HIL) experiments and aircraft tests. Most of the former research was not model based. The pressure bias control law is studied by Tang in [2], which shows reliable performance in simulation and tests. And the variable structure control and slip rate optimal control were also designed in the literatures $[3,4]$

The tire/road adhesion force model is essential for model based control laws. Early research [5] focused on the adhesion force representation, which is well-known as "Magic Formula", gives a nice approximation to experimental results of the relationship between friction coefficient and longitudinal slip. Recent research brings one kind of dynamic tire/road friction force model into ABS control, which has a potential advantage to describe closely some of the physical phenomena and to depend on parameters directly related with phenomena, called the LuGre Model. This model concerns the many-to-one mapping from velocity and other variables to adhesion coefficient ${ }^{[6]}$.

Many ABS control strategies are realized using the LuGre dynamic friction model under various road conditions. Especially, the parametric uncertainties due to road condition or/and temperature changing was considered in [7], in which one state observer was constructed to estimate internal friction state and longitudinal velocity. By utilizing LuGre dynamic friction force model, the desired maximum slip rate is estimated online. Thus, an observer-based braking controller is designed to track the desired slip rate. These models have been used successfully to identify and compensate the friction in mechanical system. In [8], a dualobserver was derived to capture different nonlinearities of friction state introduced by LuGre dynamic model, which can be used to improve the desired slip rate tracking performance. In case of possibility of entering nonrobustness, an adaptive robust controller is designed to guarantee the stability of estimation ${ }^{[9,10]}$. However, these strategies are developed without robust consideration.

In this study, the robust analysis is introduced to ABS control system. Firstly, the nonlinear fuselage and wheel models are established. The tire/road friction force is modeled by LuGre dynamic friction model considering velocity correlation, in which the unmeasurable internal friction state is estimated by dual-observer. The control target, desired slip rate, is calculated online with the help of pseudo-static distributed LuGre model. At last a robust controller for aircraft ABS is designed considering external disturbance like vertical load changing and modeling error. Simulations are implemented to verify the theoretical design.

\section{SYSTEM DYNAMICS}

\section{A. Aircraft fuselage and wheel dynamics modeling}

The aircraft ground dynamics model is shown in figure 1 , from which we can see there are lift (L), engine thrust (T), aerodynamic drag (D), gravity $(\mathrm{G})$ and landing gear forces acting on the fuselage. The landing gear forces are transferred from interaction between aircraft wheels and 
runny, including longitudinal and normal nose landing gear force $\left(\mathrm{F}_{\mathrm{ln}}\right.$ and $\left.\mathrm{F}_{\mathrm{nn}}\right)$ and left and right main landing gear forces $\left(\mathrm{F}_{\mathrm{ll}}, \mathrm{F}_{\mathrm{lr}}, \mathrm{F}_{\mathrm{nl}}\right.$ and $\left.\mathrm{F}_{\mathrm{nr}}\right)$. When only considering the longitudinal forces, we have

$$
m \dot{v}=T-D-F_{l n}-F_{l r}-F_{l l}
$$

where $\mathrm{v}$ is aircraft longitudinal speed.

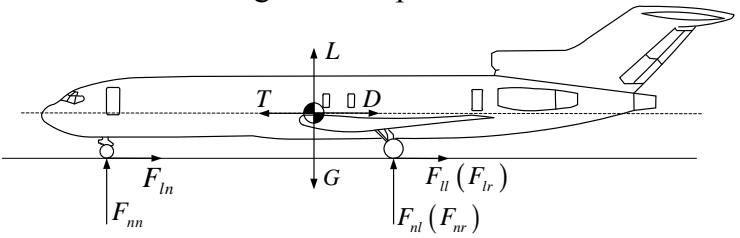

Figure. 1 Forces on the fuselage

For the sake of simple, there are some assumptions below: Assumption 1:

1) There are symmetric brake pressure signals between left and right braking system and same working conditions (runway state and temperature, etc.), thus, we have

$$
F_{x} \triangleq F_{l l}=F_{l r} ;
$$

2) There are no lateral forces or acceleration, which means that the vertical load has uniform distribution, thus, we have the same normal force

$$
F_{n} \triangleq F_{n l}=F_{n r}
$$

3) The fuselage gravity center speed $\mathrm{v}$ and aircraft wheel speed $\omega$ can be detected with sensors;

4) Longitudinal nose landing gear force $\left(F_{l n}\right)$, engine thrust and lift are neglected account for their small magnitude compared with main landing gear longitudinal force $\left(\mathrm{F}_{\mathrm{x}}\right)$ and gravity.

Remark 1: It is obviously that the system is ideally modeled and some conditions are ignored, which means that there must be model mismatch due to external disturbance and modeling error. And this term is defined as $\Delta_{\mathrm{a}}$ which will be taken into consider later in the design of robust control.

Thus, based on Assumptions 1, we have

$$
m \dot{v}=-2 F_{x}-K_{a} v^{2} \text {. }
$$

where $\mathrm{K}_{\mathrm{a}}$ is the aerodynamic drag coefficient and $\mathrm{D}=\mathrm{K}_{\mathrm{a}} \mathrm{v}^{2}$.

The aircraft wheel dynamics model is shown in figure 2 , from which we can see the brake torque $\left(u_{\tau}\right)$ is applied on the rolling wheel. The brake torque magnitude has a complicated nonlinear relationship with brake valve order signal $\left(\mathrm{P}_{\mathrm{b}}\right)$. But here we simplify it into linear relationship due to its high frequency response and weak effect on the control performance. The representation is $\mathrm{u}=\mathrm{K}_{\mathrm{b}} \mathrm{P}_{\mathrm{b}}$, and $\mathrm{K}_{\mathrm{b}}$ $=n_{p} \mu_{p} n_{h} A_{p} R_{b}$, which consists the informations of the friction faces of friction parts number $\left(\mathrm{n}_{\mathrm{p}}\right)$, friction coefficient of friction parts $\left(\mu_{p}\right)$, brake piston number $\left(n_{h}\right)$, piston area $\left(A_{p}\right)$ and friction radius $\left(\mathrm{R}_{\mathrm{b}}\right)$.

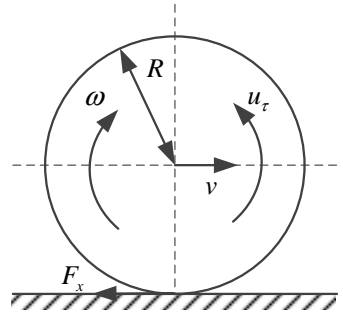

Figure. 2 Dynamics of aircraft wheel

Thus, the aircraft tire model drafted in figure 2 can be described by

$$
J \dot{\omega}=r F_{x}-K_{b} P_{b}
$$

where $J$ is rotational inertia of aircraft wheel and $r$ is the wheel rolling radius.

\section{B. Aircraft tire/road friction model}

The aircraft tire/road friction coefficient is defined by the ratio of braking force and the normal force, which can be expressed as:

$$
\mu=\frac{F_{x}}{F_{n}},
$$

where the friction coefficient $\mu$ is a complex function of the aircraft longitudinal slip and other factors, such as tire and runny conditions. The longitudinal slip is defined by:

$$
\lambda=\frac{v-r \omega}{v} \text {. }
$$

The dynamic tire/road contact friction model is described by the functions below ${ }^{[6]}$ :

$$
\begin{gathered}
\frac{d z}{d t}=v_{r}-\frac{\sigma_{0}\left|v_{r}\right|}{g\left(v_{r}\right)} z, \\
f_{x}(t)=\left(\sigma_{0} z+\sigma_{1} \frac{d z}{d t}+\sigma_{2} v_{r}\right) F_{n},
\end{gathered}
$$

with

$$
g\left(v_{r}\right)=\mu_{C}+\left(\mu_{S}-\mu_{C}\right) e^{-\left|v_{r} / v_{s}\right|^{1 / 2}}
$$

where, $\mathrm{z}$ the internal weighted mean friction state, $\mathrm{v}_{\mathrm{r}}=(\mathrm{v}-$ $r \omega)$ is the relative velocity, $f_{x}$ is the tire/road friction force model; $\sigma_{0}$ is the normalized rubber longitudinal lumped stiffness, $\sigma_{1}$ is the normalized rubber longitudinal lumped damping, $\sigma_{2}$ is the normalized viscous relative damping, $\mu_{c}$ is the normalized Coulomb friction, $\mu_{\mathrm{s}}$ is the normalized static friction $\left(\mu_{\mathrm{c}} \leq \mu_{\mathrm{s}}, \in[0,1]\right), \mathrm{v}_{\mathrm{s}}$ is the Stribeck relative velocity.

Remark 2: $\mathrm{z}$ represents the wheel bristle deflection which an essential state variable for the friction force model ${ }^{[6]}$. And the value of $\mathrm{z}$ is unknown and unmeasurable, so observers are employed to estimate the magnitude of $\mathrm{z}$, which will be studied in part III.

It is shown in [6] that the model has following finite bristle deflection property:

P1. If $|z(0)| \leq \mu_{s} / \sigma_{0}$, then $|z(t)| \leq \mu_{s} / \sigma_{0}, \forall t \geq 0$, 
P2. $\infty>\mu_{S} \geq g\left(v_{r}\right) \geq \mu_{C}>0, \forall v_{r}$.

Remark 3: $\mathbf{P 1}$ is physically intuitive and used in the subsequent controller design for a guaranteed robust performance. And it ensures that the internal friction states are bounded and that its upper bound is given by the static friction parameter and bristle stiffness.

Considering the modeling error of entire system and LuGre model compared with the actual tire/road friction force

$$
\Delta_{m}=F_{x}-f_{x}+\Delta_{a}
$$

where $\Delta_{\mathrm{a}}$ is the system modeling error defined in Remark 1.

From (4) and (5), the whole system can be rewritten as:

$$
\begin{gathered}
m \dot{v}=-2 f_{x}-K_{a} v^{2}+2 \Delta_{m}, \\
J \dot{\omega}=f_{x} r-u_{\tau}+\Delta_{m} r .
\end{gathered}
$$

\section{ROBUST CONTROLLER DESIGN}

\section{A. Control target}

The control law designed in this paper is in the purpose of making full use of the aircraft tire/road adhesion force/coefficient, which can shorten the braking distance. In the other words, a maximum deceleration is achieved with the same target. The desired slip rate $\lambda_{d}$ is corresponding to the maximum adhesion force $\mathrm{F}_{\mathrm{xmax}}$ and coefficient $\mu_{\max }$ and $\mathrm{V}_{\mathrm{rd}}$ is the desired relative speed corresponding to $\lambda_{\mathrm{d}}$ through the definition in (7). Thus, we can get the definition of braking efficiency by

$$
\eta=\frac{\int_{\lambda_{L}}^{\lambda_{H}} \mu(\lambda)}{\mu_{\text {max }}\left(\lambda_{H}-\lambda_{L}\right)}
$$

where, $\lambda_{\mathrm{H}}$ and $\lambda_{\mathrm{L}}$ are slip rate represent the first slip occurrence value and last brake releasing occurrence value. According to (13), it is known that high braking efficiency can be obtained by holding braking system working in the area around $\lambda_{\mathrm{d}}$. By solving the distributed LuGre tire/road friction model and obtain the following pseudo-static relationship between $\mu$ and $\lambda$, we have

$$
\mu=g\left(v_{r}\right)\left[1+2 \gamma \frac{g\left(v_{r}\right)}{\sigma_{0} L|\eta|}\left(e^{-\frac{\sigma_{0} L|\eta|}{2 g\left(v_{r}\right)}}-1\right)\right]+\sigma_{2} v_{r},
$$

with

$$
\eta=\frac{\lambda}{1-\lambda}, \gamma=1-\frac{\sigma_{1}|\eta|}{r \omega g\left(v_{r}\right)},
$$

where $\mathrm{L}$ is length of the tire/road contact patch.

Thus, assuming the parameters in equivalent pseudostatic model Equation (14) are known, the maximum slip rate $\lambda_{\mathrm{d}}$ corresponding to $\mu_{\max }$ can be calculated by solving it numerically. Then, $v_{\mathrm{rd}}$ is obtained for its definition. Thus, we have

$$
\frac{d v_{r}}{d t}=\frac{d v}{d t}-r \frac{d \omega}{d t} .
$$

Through linearly parameterizing the system model by substituting Equations (8), (9), (11), (12) to (15) gives

$$
\begin{aligned}
& g_{1} \frac{d v_{r}}{d t}=P_{b}-g_{2} \sigma_{0} z+g_{2} \sigma_{1} \frac{\sigma_{0}\left|v_{r}\right|}{g\left(v_{r}\right)} z \\
& -g_{2}\left(\sigma_{1}+\sigma_{2}\right) v_{r}-\frac{g_{1} K_{a}}{m} v^{2}+\Delta
\end{aligned}
$$

where, $\mathrm{g}_{1}=\mathrm{J} /\left(\mathrm{rK}_{\mathrm{b}}\right), \mathrm{g} 2=\mathrm{F}_{\mathrm{n}}\left(2 \mathrm{~J} / \mathrm{m}+\mathrm{r}^{2}\right) /\left(\mathrm{rK}_{\mathrm{b}}\right), \Delta=\Delta_{\mathrm{m}}\left(2 \mathrm{~J} / \mathrm{m}+\mathrm{r}^{2}\right)$ $/\left(\mathrm{rK}_{\mathrm{b}}\right)+\Delta_{\mathrm{n}}$.

Noting that, $g_{1}$ and $g_{2}$ are positive; $\Delta_{n}$ is the uncertain nonlinearity, so $\Delta$ represents the modeling error and uncertain nonlinearity.

Assumption 2: The modeling error and uncertain nonlinearity are bounded by a known positive constant

$$
|\Delta| \leq \delta
$$

where $\delta$ is the known bound of $\Delta$.

Obviously, the physical tire/road friction can serve limited adhesion force for the system, which can be treated as the maximum upper bound for $\delta$.

\section{B. Observers design}

Based on the aircraft tire/road friction force description in (8) and (9), we can get the system dynamics represented by (11) and (12). Considering the description of part A in this section, the control target is to track the desired relevant speed $v_{\mathrm{rd}}$ obtained online by the calculation of desired slip rate $\lambda_{\mathrm{d}}$. Thus, the entire system dynamics can be expressed in (16). Due to the unmeasurable internal friction state $\mathrm{z}$, the observer should be employed to obtain it. Considering the different nonlinear characteristics of friction state $\mathrm{z}$ in the two terms $g_{2} \sigma_{0} Z$ and $g_{2} \sigma_{1} \sigma_{0}\left|v_{r}\right| z / g\left(v_{r}\right)$ of (16), a dual-observer structure is employed here which is proposed by Tan et al in [8]. This manipulation takes more flexibility to deal with different nonlinearities in the dynamics.

Theorem 1: In the case of the absence of uncertain nonlinearities and modeling error, i.e., $\Delta=0$, suppose that the aircraft tire/road friction force is described by the model in (8) and (9), and consider the aircraft braking system equation in (11) and (12), the globally asymptotic tracking of online calculated desired slip rate $\lambda_{\mathrm{r}}$, which is corresponding to $\mu_{\max }$ trajectory, is achieved by the following nonlinear control law:

$$
\begin{aligned}
& P_{b n}=-k_{1} e+g_{2} \sigma_{0} \hat{z}_{0}-g_{2} \sigma_{1} \frac{\sigma_{0}\left|v_{r}\right|}{g\left(v_{r}\right)} \hat{z}_{1} \\
& +g_{2}\left(\sigma_{1}+\sigma_{2}\right) v_{r}+g_{1} \dot{v}_{r d}+\frac{g_{1} K_{a}}{m} v^{2}
\end{aligned}
$$

where e represents the desired relative velocity tracking error and ${ }_{0}$ and ${ }_{1}$ represent the estimates of friction state.

The estimates of internal friction state $\mathrm{z}$ are obtained by the observers below to handle the different nonlinearities: 


$$
\begin{gathered}
\frac{d \hat{z}_{0}}{d t}=v_{r}-\frac{\sigma_{0}\left|v_{r}\right|}{g\left(v_{r}\right)} \hat{z}_{0}-\gamma_{0} e, \\
\frac{d \hat{z}_{1}}{d t}=v_{r}-\frac{\sigma_{0}\left|v_{r}\right|}{g\left(v_{r}\right)} \hat{z}_{1}+\gamma_{1} \sigma_{0} \frac{\left|v_{r}\right|}{g\left(v_{r}\right)} e,
\end{gathered}
$$

where $\gamma_{0}$ and $\gamma_{1}$ are positive constants to be designed.

Proof: Base on the feedback linearization principles. With the choice of $\mathrm{P}_{\mathrm{bn}}$ in (17), we have

$$
g_{1} \frac{d e}{d t}=-k_{1} e-g_{2} \sigma_{0} \tilde{z}_{0}+g_{2} \sigma_{1} \frac{\sigma_{0}\left|v_{r}\right|}{g\left(v_{r}\right)} \tilde{z}_{1},
$$

where the modeling error and uncertain nonlinearity $\Delta=0$.

Define the following positive-semi-definite (p.s.d.) candidate Lyapunov function

$$
V_{n}=\frac{1}{2} g_{1} e^{2}+\frac{1}{2 \gamma_{0}} g_{2} \sigma_{0} \tilde{z}_{0}^{2}+\frac{1}{2 \gamma_{1}} g_{2} \sigma_{1} \tilde{z}_{1}^{2}
$$

It is worth announcing that, the observation errors in (21) can be described by (this will be utilized in the later proof)

$$
\begin{gathered}
\frac{d \tilde{z}_{0}}{d t}=-\frac{\sigma_{0}\left|v_{r}\right|}{g\left(v_{r}\right)} \tilde{z}_{0}+\gamma_{0} e, \\
\frac{d \tilde{z}_{1}}{d t}=-\frac{\sigma_{0}\left|v_{r}\right|}{g\left(v_{r}\right)} \tilde{z}_{1}-\gamma_{1} \sigma_{0} \frac{\left|v_{r}\right|}{g\left(v_{r}\right)} e,
\end{gathered}
$$

where $\tilde{z}_{0}=z-\hat{z}_{0}, \tilde{z}_{1}=z-\hat{z}_{1}$ and $\mathrm{z}$ is the unmeasurable real value of friction state.

The derivative of $V_{n}$ along trajectories in (18), (19) and (20) is

$$
\dot{V}_{n}=-k_{1} e^{2}-\frac{g_{2}}{\gamma_{0}} \frac{\sigma_{0}^{2}\left|v_{r}\right|}{g\left(v_{r}\right)} \tilde{z}_{0}^{2}-\frac{g_{2}}{\gamma_{1}} \sigma_{1} \frac{\sigma_{0}\left|v_{r}\right|}{g\left(v_{r}\right)} \tilde{z}_{1}^{2} \leq 0,
$$

With P1, the real value (z), estimation value $\left(\begin{array}{ll}0, & 1\end{array}\right)$ and estimation errors $\left(\tilde{z}_{0}, \tilde{z}_{1}\right)$ of friction state are bounded. Based on the definition of $\mathrm{v}_{\mathrm{rd}}$, physical limitation of $\mathrm{v}$ and $\mathrm{v}_{\mathrm{r}}$, the boundedness of tracking error e is obvious. Due to the representations of $\mathrm{g}_{2}$ and $\mathrm{g}\left(\mathrm{v}_{\mathrm{r}}\right)$, the constants $\sigma 0, \sigma 1$ and $\sigma 2$, the boundedness of controller is apparent from the structure in (17).

The boundedness of all internal variables and constants are proved. Thus, the derivative of the relative velocity tracking error e is also bounded. By (24), we have e $\in \mathrm{L}_{2}$. Combining the fact that $\mathrm{e} \in \mathrm{L}_{\infty}$ and $\dot{\mathrm{e}} \in \mathrm{L}_{\infty}$ and utilizing the Barbalat's lemma, we conclude that, $\mathrm{e} \rightarrow 0$, as $\mathrm{t} \rightarrow \infty$. Thus, by the definition of e and $\lambda$ in (7), we have $\lambda \rightarrow \lambda_{\mathrm{d}}$, as $\mathrm{t} \rightarrow \infty$.

\section{Robust controller design}

To avoid entering the unstable estimation of the unmeasurable friction states $\mathrm{z}$, the following robust observers with projection-type modifications which are based on the observers design in part $\mathrm{B}$

$$
\begin{gathered}
\frac{d \hat{z}_{0}}{d t}=\operatorname{Proj}_{z_{0}}\left(v_{r}-\frac{\sigma_{0}\left|v_{r}\right|}{g\left(v_{r}\right)} \hat{z}_{0}-\gamma_{0} e\right), \\
\frac{d \hat{z}_{1}}{d t}=\operatorname{Proj}_{z_{1}}\left(v_{r}-\frac{\sigma_{0}\left|v_{r}\right|}{g\left(v_{r}\right)} \hat{z}_{1}+\gamma_{1} \sigma_{0} \frac{\left|v_{r}\right|}{g\left(v_{r}\right)} e\right) .
\end{gathered}
$$

The projection mapping $\operatorname{Proj}_{\zeta}(*)$ has similar structure with Sastry in [11],

$$
\operatorname{Prog}_{\zeta}(*)=\left\{\begin{array}{l}
0 \text { if } \hat{\zeta}=\zeta_{\text {max }} \text { and } *>0 \\
0 \text { if } \hat{\zeta}=\zeta_{\text {min }} \text { and } *<0 \\
* \text { otherwise }
\end{array}\right.
$$

where $\zeta$ is a symbol that can be replaced by $z_{0}$ and $z_{1}$.

P2. The projection mapping has the following properties:

$$
\begin{gathered}
\tilde{z}_{0}\left\{\operatorname{Proj}_{z_{0}}\left(v_{r}-\frac{\sigma_{0}\left|v_{r}\right|}{g\left(v_{r}\right)} \hat{z}_{0}-\gamma_{0} e\right)-\right. \\
\left.\left(v_{r}-\frac{\sigma_{0}\left|v_{r}\right|}{g\left(v_{r}\right)} \hat{z}_{0}-\gamma_{0} e\right)\right\} \geq 0 \\
\tilde{z}_{1}\left\{\operatorname{Proj}_{z_{1}}\left(v_{r}-\frac{\sigma_{0}\left|v_{r}\right|}{g\left(v_{r}\right)} \hat{z}_{1}+\gamma_{1} \sigma_{0} \frac{\left|v_{r}\right|}{g\left(v_{r}\right)} e\right)-\right. \\
\left.\left(v_{r}-\frac{\sigma_{0}\left|v_{r}\right|}{g\left(v_{r}\right)} \hat{z}_{1}+\gamma_{1} \sigma_{0} \frac{\left|v_{r}\right|}{g\left(v_{r}\right)} e\right)\right\} \geq 0
\end{gathered}
$$

Lemma 1: Considering the aircraft braking system described in (11), (12) and tire/road friction force in the form of (8) and (9) with uncertain nonlinearities, i.e., $\Delta \neq 0$, we can find the nonlinear robust control law in the following structure

$$
P_{b}=P_{b n}+P_{b r}
$$

where $P_{b n}$ is shown in (17). There exists a nonlinear robust control term $\mathrm{P}_{b r}$, in a form which satisfies the following relationships:

$$
s=e\left(P_{b r}-g_{2} \sigma_{0} \tilde{z}_{0}+g_{2} \sigma_{1} \frac{\sigma_{0}\left|v_{r}\right|}{g\left(v_{r}\right)} \tilde{z}_{1}+\Delta\right) \leq \varepsilon,
$$

where $\mathrm{P}_{b r}$ is synthesized to attenuate the effect of the uncertain nonlinearities and modeling error, and $\varepsilon$ is a constant to be designed. And

$$
e P_{b r} \leq 0 \text {. }
$$

Proof: Based on last part of the proof for Theorem 1, the internal friction state and parameters have the properties of boundedness. Thus, the robust control term $\mathrm{P}_{\mathrm{br}}$ here always exists. Using the similar technique in $\mathrm{Yao}^{[10]}$, we choose smooth bounding function $\mathrm{h}$ satisfying: 


$$
h \geq g_{2}^{2} \sigma_{0}^{2} z_{r}^{2}\left(1+\sigma_{1}^{2} \frac{\left|v_{r}\right|^{2}}{g^{2}\left(v_{r}\right)}\right)+\delta^{2},
$$

where $\mathrm{z}_{\mathrm{r}}=\left|\mathrm{z}_{\max }-\mathrm{z}_{\min }\right|$ (also exists $\mathrm{z}_{\mathrm{r}}=\left|\begin{array}{l}0 / 1 \max \\ -\end{array} \quad 0 / 1 \min \right|$ by projection mapping in (25) and (26)).

And we choose the robust control term $\mathrm{P}_{\mathrm{br}}$ in a form as

$$
P_{b r}=\frac{3}{4 \varepsilon} h \text {. }
$$

Thus, it is easily to have from (31) that

$$
s \leq|e| P_{b r}+|e| g_{2} \sigma_{0} z_{r}+|e| g_{2} \sigma_{1} \frac{\sigma_{0}\left|v_{r}\right|}{g\left(v_{r}\right)} z_{r}+|e| \delta \text {. }
$$

Substituting $\mathrm{P}_{\mathrm{br}}$ into (35), we have

$$
\begin{aligned}
& s \leq-\left(\frac{\sqrt{3} g_{2} \sigma_{0} z_{r}}{2 \sqrt{\varepsilon}}|e|-\sqrt{\frac{\varepsilon}{3}}\right)^{2}- \\
& \left(\frac{\sqrt{3} g_{2} \sigma_{1} \sigma_{0} z_{r}\left|v_{r}\right|}{2 \sqrt{\varepsilon} g\left(v_{r}\right)}|e|-\sqrt{\frac{\varepsilon}{3}}\right)^{2}-. \\
& \left(\frac{\sqrt{3} \delta}{2 \sqrt{\varepsilon}}|e|-\sqrt{\frac{\varepsilon}{3}}\right)^{2}+\varepsilon \leq \varepsilon
\end{aligned}
$$

Thus the inequation in (31) is proved. According to the definition of e and $\mathrm{P}_{\mathrm{br}}$, it is easily to have that (32) is satisfied. Theorem 2: Considering the aircraft braking system described in (11), (12) and tire/road friction force in the form of (8) and (9) with uncertain nonlinearities, i.e., $\Delta \neq 0$, we can find the nonlinear robust control law in the same structure as in (30), which is rewritten here as following,

$$
P_{b}=P_{b n}+P_{b r},
$$

where $P_{b n}$ is given in (17), the aircraft tire/road internal friction state observers are given in (25) and (26); $\mathrm{P}_{\mathrm{br}}$ represents the robust control term in the form of (34), we have,

1) All the signals present in system are bounded. Furthermore, the candidate Lyapunov function in (39) satisfies:

$$
V_{r}(t) \leq e^{-\frac{2 k_{1}}{g_{1}} t} V_{r}(0)+\frac{\varepsilon g_{1}}{2 k_{1}}\left(1-e^{-\frac{2 k_{1}}{g_{1}} t}\right),
$$

2) If after a finite time $t_{0}$, in absence of any uncertainties, i.e., $\Delta=0$, by utilizing the proposed controller in (30), the online calculated desired slip rate $\lambda_{\mathrm{d}}$, which is corresponding to the $\mu_{\max }$, can be achieved.

Proof: Applying $\mathrm{P}_{\mathrm{b}}$ synthesized in the form of (30) into the proposed system, we have

$g_{1} \frac{d e}{d t}=-k_{1} e-g_{2} \sigma_{0} \tilde{z}_{0}+g_{2} \sigma_{1} \frac{\sigma_{0}\left|v_{r}\right|}{g\left(v_{r}\right)} \tilde{z}_{1}+\Delta+P_{b r}$,

Considering the following p.s.d. candidate Lyapunov function,

$$
V_{r}=\frac{1}{2} g_{1} e^{2} .
$$

The derivative of $\mathrm{V}_{\mathrm{r}}$ along trajectories in (25), (26) and (38) is

$$
\dot{V}_{r}=-k_{1} e^{2}+e\left(-g_{2} \sigma_{0} \tilde{z}_{0}+g_{2} \sigma_{1} \frac{\sigma_{0}\left|v_{r}\right|}{g\left(v_{r}\right)} \tilde{z}_{1}+\Delta+P_{b r}\right),
$$

Considering (31) proved in Lemma 1 and the structure of $V_{r}$, we have

$$
\dot{V}_{r} \leq-k_{1} e^{2}+\varepsilon \leq-\frac{2 k_{1}}{g_{1}} V_{r}+\varepsilon .
$$

Utilizing comparison lemma, we have the condition in (37). Thus, $\mathrm{V}_{\mathrm{r}}(\mathrm{t})$ exponentially decays and is ultimately bounded. With the help of Assumption 2, the relative velocity $\mathrm{v}_{\mathrm{r}}$ tracking error is bounded. Account for the $\mathrm{P} 1$, the internal friction states has the properties of boundedness. Through the projection mapping in (25) and (26), the estimates of state 0 and 1 are bounded by $\mu_{\mathrm{s}} / \sigma_{0}$. Thus the braking pressure input $\mathrm{P}_{\mathrm{b}}$ is bounded.

Define the same p.s.d. candidate Lyapunov function $V_{n}$ as in (21). By substituting (22) and (23), the derivative of $V_{n}$ along trajectories in (25), (26) and (38) is

$$
\begin{aligned}
& \dot{V}_{n}=-k_{1} e^{2}+e P_{b r}-g_{2} \sigma_{0} \tilde{z}_{0}\left(e+\frac{\dot{\hat{z}}_{0}}{\gamma_{0}}\right)+ \\
& g_{2} \sigma_{1} \tilde{z}_{1}\left(\frac{\sigma_{0}\left|v_{r}\right|}{g\left(v_{r}\right)} e-\frac{\dot{\hat{z}}_{1}}{\gamma_{r}}\right)+\frac{g_{2} \sigma_{0} \tilde{z}_{0} \dot{z}}{\gamma_{0}}+\frac{g_{2} \sigma_{1} \tilde{z}_{1} \dot{z}}{\gamma_{1}} \\
& \quad=-k_{1} e^{2}+e P_{b r}-\frac{g_{2} \sigma_{0} \tilde{z}_{0}}{\gamma_{0}}\left\{\dot{\hat{z}}_{0}-\left(v_{r}-\frac{\sigma_{0}\left|v_{r}\right|}{g\left(v_{r}\right)} \hat{z}_{0}-\gamma_{0} e\right)\right\} \\
& -\frac{g_{2} \sigma_{1} \tilde{z}_{1}}{\gamma_{1}}\left\{\dot{z}_{1}-\left(v_{r}-\frac{\sigma_{0}\left|v_{r}\right|}{g\left(v_{r}\right)} \hat{z}_{1}+\gamma_{1} \frac{\sigma_{0}\left|v_{r}\right|}{g\left(v_{r}\right)} e\right)\right\}+ \\
& \frac{g_{2} \sigma_{0} \tilde{z}_{0}}{\gamma_{0}}\left(\dot{z}-v_{r}+\frac{\sigma_{0}\left|v_{r}\right|}{g\left(v_{r}\right)} \hat{z}_{0}\right)+\frac{g_{2} \sigma_{1} \tilde{z}_{1}}{\gamma_{1}}\left(\dot{z}-v_{r}+\frac{\sigma_{0}\left|v_{r}\right|}{g\left(v_{r}\right)} \hat{z}_{1}\right)
\end{aligned}
$$

Substituting the definition of $\dot{z}$ in (8), (28) and (29) in P2 and (32) in Lemma 1, combining the positive parameters in $\mathrm{V}_{\mathrm{n}}$, we have

$$
\dot{V}_{n}=-k_{1} e^{2}-\frac{g_{2}}{\gamma_{0}} \frac{\sigma_{0}^{2}\left|v_{r}\right|}{g\left(v_{r}\right)} \tilde{z}_{0}^{2}-\frac{g_{2}}{\gamma_{1}} \frac{\sigma_{0}^{2}\left|v_{r}\right|}{g\left(v_{r}\right)} \tilde{z}_{1}^{2} \leq-k_{1} e^{2} .
$$

According to (42), we have e $\in \mathrm{L}_{2}$. Combining the fact that $\mathrm{e} \in \mathrm{L}_{\infty}$ and $\dot{\mathrm{e}} \in \mathrm{L}_{\infty}$ and utilizing the Barbalat's lemma, we conclude that, $\mathrm{e} \rightarrow 0$, as $\mathrm{t} \rightarrow \infty$. Thus, by the definition of e and $\lambda$ in (7), we have $\lambda \rightarrow \lambda_{d}$, as $t \rightarrow \infty$, which means the $\mu_{\max }$ is achieved correspondingly. 


\section{Simulation RESUlTS}

Simulations have been performed based on the aircraft system described by aircraft braking system in (8), (9) and aircraft tire/road friction force in (11), (12). The friction parameters and system parameters are given in TABLE 1 ,

TABLE I.

SIMULATION PARAMETERS

\begin{tabular}{|l|l|l|l|l|l|}
\hline Name & Value & Units & Name & Value & Units \\
\hline$\sigma_{0}$ & 1 & $1 / \mathrm{m}$ & $\mathrm{r}$ & 0.323 & $\mathrm{~m}$ \\
\hline$\sigma_{1}$ & 0.1487 & $\mathrm{~s} / \mathrm{m}$ & $\mathrm{m}$ & 3720 & $\mathrm{Kg}$ \\
\hline$\sigma_{2}$ & 0.0038 & $\mathrm{~s} / \mathrm{m}$ & $\mathrm{J}$ & 2.603 & $\mathrm{Kg} \mathrm{m}^{2}$ \\
\hline$\mu_{\mathrm{C}}$ & 0.5 & - & $\mathrm{g}$ & 9.8 & $\mathrm{~m} / \mathrm{s}^{2}$ \\
\hline$\mu_{\mathrm{S}}$ & 0.9 & - & $\mathrm{v}$ & 50.94 & $\mathrm{~m} / \mathrm{s}$ \\
\hline $\mathrm{V}_{\mathrm{s}}$ & 12.5 & $\mathrm{~m} / \mathrm{s}$ & $\mathrm{Z}_{\mathrm{r}}$ & 1.8 & $\mathrm{~N} / \mathrm{m}$ \\
\hline
\end{tabular}

The initial aircraft speed and wheel linear speed are the same $(50.94 \mathrm{~m} / \mathrm{s})$. Since the $0 \mathrm{~s}$, a braking pressure is applied by the braking actuator on friction parts. The desired slip rate is calculated online, and correspondingly the relative velocity is given to the robust controller as the tracking signal. Noting that, a band-limited white noise signal with power of 0.0001 is injected into the desired slip rate signal from 0 s to $7 \mathrm{~s}$ to simulate external disturbance. The initial values of all integrators are 0 . The simulation results show that, the performances of two observers are effective and the system response (slip rate etc.) converge to the real/desired values fast and then recover to their normal values after disturbance.

Figure 3 shows the real value captured from object model and the estimation values of the dual-observer and estimation errors are shown in figure 4. Figure 5 shows the braking pressure order sent to the braking actuator. Figure 6 shows the slip rate calculated by the aircraft and wheel velocity (shown in figure 7) and the desired value calculated online with external disturbance. Once the target of tracking the online calculated desired slip rate is achieved, the maximum adhesion force and deceleration are achieved as well. So we can conclude that, according to the result in figure 6 , the desired brake performance is achieved by applying brake pressure signal in figure 5.
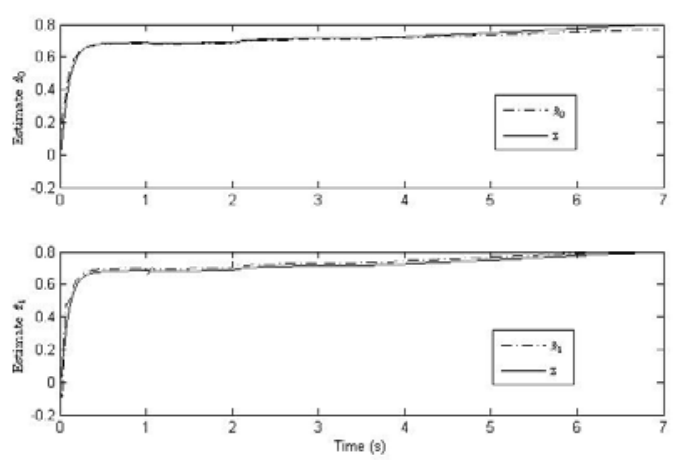

Figure. 3 Estimated and real internal friction state values.
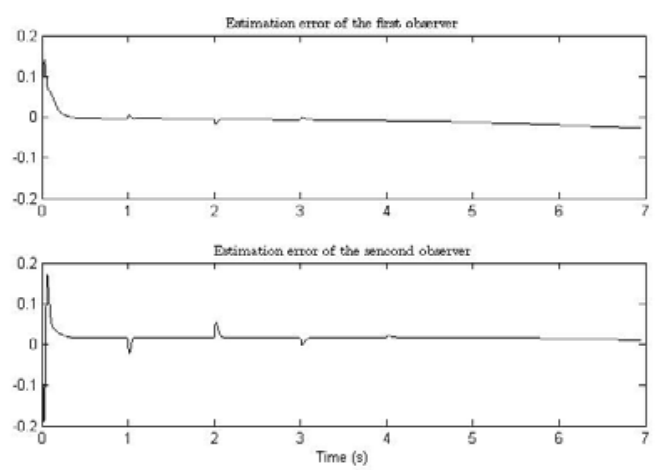

Figure. 4 Estimation errors of dual-observer

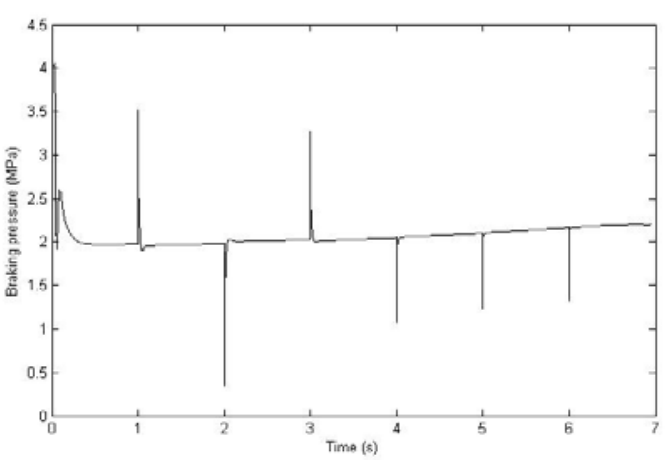

Figure. 5 Braking pressure signal applied to the actuator

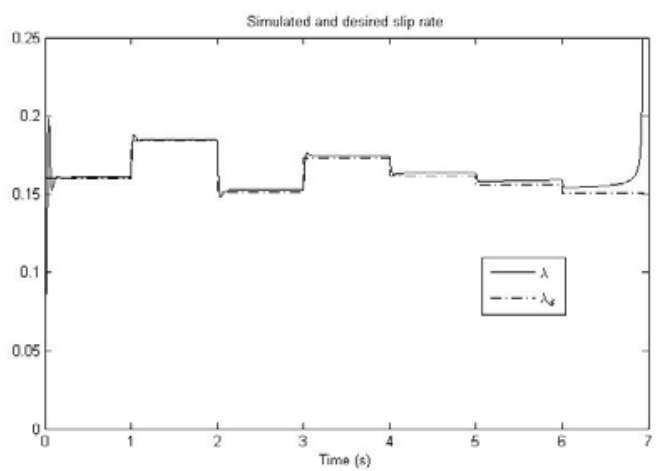

Figure. 6 Aircraft system simulated and desired slip rate values.

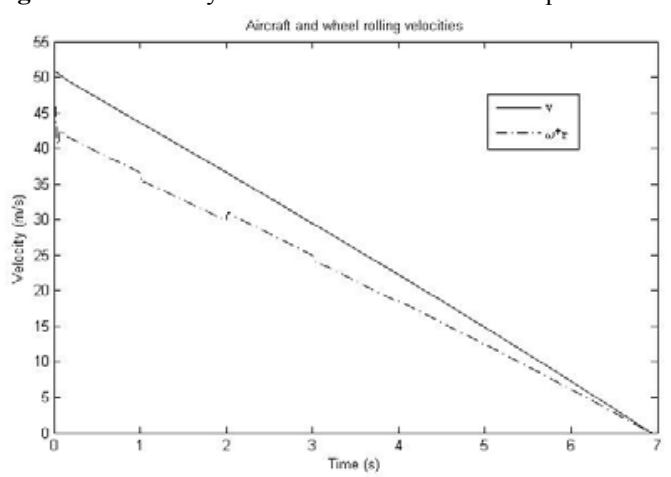

Figure. 7 Aircraft and linear wheel velocities. 


\section{CONCLUSION}

In this study, a nonlinear robust controller is proposed based on the LuGre dynamic friction force model. The braking pressure controller is determined by the estimates of unmeasurable internal friction states. A dual-observer is employed to estimate unmeasurable states with the projection mapping and a serious of boundedness assumptions in order not to obtain unstable estimates. The simulation results show that the aircraft can be stopped effectively with a near maximum slip rate or deceleration by applying the proposed controller. The asymptotic convergence of the estimated states is also proved theoretically and by simulation results.

\section{REFERENCES}

[1] Wang Ji sen. Nonlinear control theory and application to aircraft antiskid system. Xi'an: Northwestern Poly technical University, 2001(in Chineses).

[2] Tang Chuanye. Simulation studies on aircraft anti-skid braking system. Xi'an: Northwestern Poly technical University, 2007(in Chineses).

[3] Li Yuren, Ma Ruiqing, Xue Jing, Xie Lili. Improving vriable structure control of aircraft. Journal of Northwestern Poly technical University, 2008,26(6):752-754(in Chineses)
[4] Tian Guanlai, Xie Lili, Yue Kaixian, Chang Shunkong. Study on optimal control method of an aircraft anti-skid braking system based on slip-ratio. Acta Aeronautica et Astronautica Sinica, 2005, 26(4):461-464(in Chineses).

[5] Bakker, E., Nyborg, L. and Pacejka, H.B., Tyre modelling for use in vehicle dynamic studies. SAE Paper. No. 870421, 1987.

[6] C. Canudas de Wit, M. L. Petersen, A. Shiriaev. A new observer for tire/road distributed contact friction. Conference on Decision and Control, Hawaii, USA, 2003.

[7] J. Yi, L. Alvarez, X. Claeys, R. Horwitz. Adaptive emergency braking control in automated highway system using a dynamic tire/road friction model. Proccedings of 39th IEEE Conference of Decision Control, Sydney, Australia, 2000, pp.456-461

[8] Yaolong Tan, Ioannis Kanellakopoulos. Adaptive nonlinear friction compensation with parametric uncertainties. Proceeding of the American Control Conference. San Diego: 1999,pp.2511-2513.

[9] P. Tomei, Robust adaptive friction compensation for tracking control of robot manipulators, IEEE Tran. on Auto. Cont., 45, pp 2164 2169, 2000.

[10] B. Yao, M. Tomizuka, Adaptive robust control of SISO non-linear systems in a semi-strict feedback form, Automatica, 33, pp. $893-900$, 1997.

[11] Sastry S, Bodson M. Adaptive control: stability, convergence and robustness. Englewood Cliffs, NJ 07632, USA: Prentice Hall, Inc, 1989. 